\title{
INCIDÊNCIA DE PERDA DE PRENHEZ ATÉ O 50 DIA EM ÉGUAS QUARTO DE MILHA
}

\author{
INCIDENCE OF PREGNANCY LOSS UNTIL THE $50^{\text {th }}$ DAY \\ IN QUARTER HORSE MARES
}

\author{
Marcelo Barbanti Duarte ${ }^{1}$ Rogério Chaves Vieira ${ }^{2}$ \\ Frederico Ozanan Carneiro e Silva ${ }^{2}$
}

\section{RESUMO}

Este estudo teve por objetivo avaliar a incidência de perda de prenhez considerando os periodos cronológicos de sua ocorrência, status reprodutivo e diferentes faixas etárias. Durante os anos hipicos de 1998/1999 e 1999/2000 (setembro a janeiro) foram monitoradas as gestações de 445 animais da raça Quarto de Milha, com idades de 3 a 20 anos, compreendendo potras de primeira cobrição, éguas falhadas e éguas paridas. Procedeu-se o diagnóstico de gestação por ultra-sonografia no $11^{\circ}$ dia pós-ovulação, repetindo-se os exames aos 20,30, 40 e 50 dias de prenhez. As éguas foram separadas em grupos de I a IV, correspondentes às perdas de prenhez entre os dias 11 e 20, 21 e 30,31 e 40, e 41 e 50, respectivamente. $O$ indice total de perdas foi de 8,53\% (38/445), sendo de 5,39\% (24/445); 1,57\% (7/445); $1,12 \%(5 / 445)$ e $0,45 \%$ (2/445) para os grupos I, II, III e IV, respectivamente. A única e significativa incidência $(P<0,05)$ ocorreu no grupo I $(63,15 \% ; 24 / 38)$, indicando que o período entre a segunda e terceira semanas é o mais suscetível à perda precoce de prenhez. Com relação ao status reprodutivo e faixas etárias, não se evidenciaram diferenças significativas $(P>0,05)$ nas perdas.

Palavras-chave: perda de prenhez, morte embrionária, eqüino, égua.

\section{SUMMARY}

The objective of this study was to evaluate the incidence of pregnancy loss, considering the periods of occurrence, reproductive status, as well as the different age groups. During the breeding seasons of 1998/1999 and 1999/2000 (September to January) the gestations of 445 Quarter Horse mares with ages from 3 to 20 years were monitored, comprehending maiden, barren and lactating. Pregnancy diagnostic was performed by ultrasonography at the $11^{\text {th }}$ day after ovulation and repeated on days 20,30, 40 and 50. The mares were distributed in groups from $I$ to $I V$, corresponding to pregnancy losses between days 11 to 20,21 to 30,31 to 40 and 41 to 50, respectively. The overall pregnancy loss rate was $8.53 \%$ (38/445), being of 5.39\% (24/445); $1.57 \%$ (7/445); $1.12 \%(5 / 445)$ and $0.45 \%(2 / 445)$ for groups $I$, II, III and IV, respectively. The unique and significant incidence $(P<0.05)$ occurred in group I $(63.15 \%, 24 / 38)$, showing that the time between the second and third weeks is the most susceptible to pregnancy loss. The loss rates were not significantly different $(P>0.05)$ concerning reproductive status and age groups.

Key words: pregnancy loss, embryonic death, equine, mare.

\section{INTRODUÇ̃̃̃O}

A perda da prenhez é um dos principais fatores de subfertilidade na espécie eqüina. Tanto a fase da gestação em que esta ocorre, quanto a incidência das perdas, relacionam-se diretamente com a saúde do trato reprodutivo (GINTHER $\boldsymbol{e t}$ al., 1985), variando ainda, entre diversos fatores, em função da idade das éguas (BALL $\boldsymbol{e t}$ al., 1986), condição nutricional do rebanho sob estudo (WOODS et al., 1987), intervalo de diagnóstico e método de determinação da prenhez ( McKINNON et al., 1988).

Registros encontrados na literatura indicam variação de 5 a $45 \%$ nos índices de perdas (BALL, 1993), refletindo diferenças entre criatórios

\footnotetext{
${ }^{1}$ Médico Veterinário, Pós-graduando do Curso de Mestrado em Ciências Veterinárias (Clínica e Cirurgia). Faculdade de Medicina Veterinária, Universidade Federal de Uberlândia (FAMEV-UFU), Av. Pará, 1720, 38400-462, Uberlândia, MG. E-mail: mdduarte@zaz.com.br. Autor para correspondência.

${ }^{2}$ Professor Titular, Doutor, FAMEV, UFU.
} 
e dificuldades de diagnóstico. A ultra-sonografia, utilizada mais intensamente em veterinária a partir da década de 80 , tem aumentado a eficácia dos exames clínicos, possibilitando detectar vesículas embrionárias com 10 ou 11 dias pós-ovulação, e observar sinais precisos sobre a evolução e subseqüente perda da prenhez (PALMER \& DRIANCOURT, 1980).

Os índices de morte do conceptus no início da gestação são mais elevados quando comparados com estádios mais avançados (GINTHER $\boldsymbol{e}$ al., 1985). Segundo esses autores tal fenômeno deve-se, provavelmente, à inadequação do meio ambiente uterino por ocasião da chegada do embrião ao útero, entre o $5^{\circ}$ e $6^{\circ}$ dia, bem como à falta de mobilidade do mesmo entre o $11^{\circ}$ e $15^{\circ}$ dia, com conseqüente ausência do reconhecimento maternal da prenhez. BLUE (1981), a partir do pressuposto de que aproximadamente $66 \%$ de abortos espontâneos em humanos, entre 21 e 49 dias, são devidos à anomalias cromossômicas (BOUIE \& BOUIE, 1976), postulou que também na espécie eqüina defeitos no embrião seriam responsáveis por relevante parcela das perdas embrionárias observadas no início da gestação.

No Brasil, LOPES et al. (1993) investigaram a morte embrionária em éguas Quarto de Milha, Puro Sangue Inglês e Mangalarga Paulista e assinalaram perdas de $13,28 \%$ para o período de 10 a 30 dias. Similarmente, FERREIRA et al. (1999) registraram proporções mais elevadas de perdas na fase inicial da gestação em animais da raça Campolina. De um total de 103 éguas prenhes, estes autores verificaram morte embrionária em 7 animais $(6,83 \%)$, sendo que, em três destes, as perdas ocorreram entre 15 e 20 dias.

WOODS et al. (1987) constataram que os índices de perda embrionária não diferiram significativamente entre os grupos de éguas cobertas no cio do potro e no cio subseqüente, os quais foram de $11 \%(18 / 157)$ e $12 \%(24 / 203)$, respectivamente. Para as éguas consideradas virgens, falhadas e paridas, os índices de perda de prenhez foram de $12 \%, 14 \%$ e $14 \%$, respectivamente. CHEVALIERCLÉMENT (1989) monitorou as gestações de éguas Puro Sangue Inglês virgens, falhadas e paridas, encontrando índices de perdas para essas categorias de respectivamente $4,5 \%, 5,4 \%$ e $6,5 \%$.

Este estudo objetivou avaliar os índices de perdas de prenhez em função dos períodos cronológicos de sua ocorrência, status reprodutivo e diferentes faixas etárias em éguas Quarto de Milha criadas no município de Uberaba, MG.

\section{MATERIAL E MÉTODOS}

O trabalho foi realizado em quatro horas no município de Uberaba, MG, durante os anos hípicos de 1998/1999 e 1999/2000 (setembro a janeiro). Foram monitoradas as gestações de 445 éguas Quarto de Milha, com idades variando de 3 a 20 anos, mantidas em piquetes de grama cost-cross (Cynodon dactilon), recebendo por cabeça, diariamente, uma suplementação de dois quilos de ração que continha $15 \%$ de proteína bruta e mistura mineral à vontade no cocho.

Para avaliação das perdas de prenhez em função do status reprodutivo, foram constituídos três grupos de animais, compreendendo 369 éguas paridas, 58 potras de primeira cobrição e 18 éguas falhadas da estação de monta anterior. A partir do segundo dia de sobreparto, as éguas eram rufiadas e monitoradas via retal quanto aos sinais clínicos de cio e ovulação. Classificou-se o cio de acordo com sua ocorrência em cio do potro e cio subseqüente. Foi considerado cio do potro quando este se iniciou até 14 dias pós-parto, como preconizado por DU PLESSIS (1964), e cio subseqüente aquele manifestado logo em seguida. As éguas eram submetidas a rigoroso exame ultra-sonográfico, sendo liberadas para inseminação apenas aquelas que, no quinto dia pós-parto, não mais evidenciavam presença de líquido no útero. Nas éguas que persistiam com líquido efetuaram-se diariamente lavagens uterinas com solução fisiológica $\mathrm{NaCl}$ $0,9 \%$, seguidas da aplicação intravenosa de $1,5 \mathrm{~m} \ell$ (15 UI) de oxitocina ${ }^{\mathrm{a}}$, até completo desaparecimento do líquido.

Foram retirados deste estudo, por ocasião dos exames ultra-sonográficos, os animais com formações císticas endometriais, assim como aqueles que, no decorrer do período de monta, apresentaram ovulações duplas, os quais foram incluídos em outro experimento. Antes e durante as estações de monta, fez-se correção cirúrgica nos indivíduos que apresentavam alterações de angulação e fechamento vulvar, bem como lacerações de períneo. As éguas em cio foram inseminadas com sêmen fresco cada 48 horas até a ovulação, a partir da identificação de um folículo $\geq$ $35 \mathrm{~mm}$ de diâmetro, contendo a dose inseminante cerca de $1,0 \quad \mathrm{X} \quad 10^{9}$ espermatozóides viáveis. Considerou-se o momento da ovulação como dia zero.

Após o diagnóstico positivo de prenhez, efetuado no $11^{\circ}$ dia, repetiram-se os exames nos dias $20,30,40$ e 50 . Registrou-se a tonicidade e 
ecogenicidade do útero, presença de fluido e pregas endometriais, além das características pertinentes à integridade da vesícula, ou seja, mobilidade, formato e batimento cardíaco do embrião. Considerou-se perda da prenhez ao constatar-se ausência de vesícula embrionária nas éguas anteriormente diagnosticadas como gestantes. Os animais foram alocados em grupos de I a IV, correspondentes às perdas nos períodos de 11 a 20, 21 a 30, 31 a 40 e 41 a 50 dias, respectivamente. Para comparar as perdas em função das diferentes faixas etárias, agruparamse as éguas por idade de 3 a 5, 6 a 8, 9 a 11 e acima de 12 anos, respectivamente.

As proporções de perdas por períodos de gestação, status reprodutivo e faixas etárias foram analisadas pelo teste de Qui-quadrado $\left(\chi^{2}\right)$ em nível de $5 \%$ de probabilidade.

\section{RESULTADOS E DISCUSSÃO}

Analisando as perdas de prenhez nos períodos cronológicos, verificou-se única e significativa $(\mathrm{P}<0,05)$ incidência no Grupo I, entre 11 e 20 dias de gestação (Tabela 1), resultado que pode ser explicado por uma provável adversidade de meio ambiente uterino, ou mesmo de oviduto (GINTHER et al., 1985; BALL \& WOODS, 1987). Ainda que de modo especulativo, esse resultado pode também ser explicado, em parte, por uma possível ocorrência de anomalias cromossômicas e defeitos morfológicos no embrião (BOUIE \& BOUIE, 1976; BLUE, 1981). Contudo, um efeito negativo de anomalias cromossômicas advindas da senilidade de gametas (BLUE, 1981) poderia ser afastado, uma vez que, pelo controle folicular, as éguas eram inseminadas com sêmen fresco próximo à ovulação.

A incidência total de perdas de prenhez observada neste trabalho, de $8,53 \%$ (38/445) para o período de 11 a 50 dias (Tabela 1), é inferior à relatada por WOODS et al. (1987), os quais, ao avaliarem 559 gestações não gemelares, constataram

Tabela 1 - Índices de perdas de prenhez em diferentes períodos da gestação.

\begin{tabular}{lrc}
\hline Períodos da gestação & $\mathrm{N}^{\mathrm{o}}$ de perdas/prenhez & $\mathrm{N}^{\mathrm{o}}$ de perdas/perdas totais \\
\hline Grupo I (11-20 dias) & $24 / 445(5,39 \%)$ & $24 / 38(63,16 \%)^{\mathrm{a}}$ \\
Grupo II (21-30 dias) & $7 / 445(1,57 \%)$ & $7 / 38(18,42 \%)^{\mathrm{b}}$ \\
Grupo III (31-40 dias) & $5 / 445(1,12 \%)$ & $5 / 38(13,16 \%)^{\mathrm{b}}$ \\
Grupo IV (41-50 dias) & $2 / 445(0,44 \%)$ & $2 / 38(5,26 \%)^{\mathrm{b}}$ \\
Total & $38 / 445(8,53 \%)$ & $38 / 38(100,0 \%)$
\end{tabular}

$\left({ }^{a}, \mathrm{~b}\right)$ letras distintas na coluna diferem estatisticamente pelo teste de $\chi^{2}$ em nível de $5 \%$.
$12,52 \%$ de perdas (70/559) entre 14 e 56 dias. Também FERREIRA et al. (1999) e LOPES $\boldsymbol{e t}$ al. (1993) relataram incidência mais elevada de perdas comparada à observada neste trabalho. Essas diferenças devem estar relacionadas à heterogeneidade dos animais investigados pelos autores supracitados, no que diz respeito à saúde do trato reprodutivo e idade dos animais, não podendo ser descartada uma possível influência da variabilidade existente na assistência clínica veterinária recebida pelos diversos haras.

Os resultados dos exames ultrasonográficos efetuados neste estudo, por ocasião do diagnóstico das perdas de prenhez, são semelhantes aos relatados por BALL (1993) e FERREIRA $\boldsymbol{e t}$ al. (1999), ou seja, notou-se a presença de pregas endometriais típicas da fase estrogênica, acompanhadas de hipoecogenicidade ou mesmo ausência do corpo lúteo no $20^{\circ}$ dia, além de subdesenvolvimento do embrião e ausência de batimentos cardíacos em torno do $30^{\circ}$ dia. Foi observado que as éguas com vesícula embrionária no $30^{\circ}$ dia e cujos embriões denotavam ausência de batimentos cardíacos, ainda mantinham o tônus uterino, ocorrência também citada por GINTHER et al. (1985). Porém, a ausência ou diminuição do tônus uterino nesses animais ficou evidenciada no exame seguinte, efetuado no $40^{\circ}$ dia, refletindo uma provável diminuição nos níveis de progesterona. Embora não existam informações definitivas sobre as concentrações ideais de progesterona necessárias ao desenvolvimento da prenhez, concentrações plasmáticas inadequadas têm sido associadas à morte embrionária (DOUGLAS et al., 1985; GINTHER et al., 1985). Nesse sentido, DOUGLAS et al. (1985) propuseram que níveis de progesterona abaixo de $2,5 \mathrm{ng} / \mathrm{m} \ell$ em torno do $12^{\underline{0}}$ dia indicam insuficiência lútea e inviabilidade da gestação. BALL \& WOODS (1987) citam que, no caso de instituir-se uma suplementação com esse hormônio, pode ocorrer prolongamento de uma prenhez já condenada, adiando-se assim o retorno ao cio e nova cobertura do animal. No presente estudo, não foram efetuadas dosagens de progesterona que poderiam contribuir para elucidar as perdas de prenhez.

Os índices de perdas não diferiram estatisticamente $(\mathrm{P}>0,05)$ entre éguas falhadas, potrancas e paridas (Tabela 2), à semelhança dos resultados de WOODS et al. (1987) e CHEVALIER-CLÉMENT (1989), que da mesma forma não constataram diferenças significativas nos índices de perdas em função do status reprodutivo. A incidência de perdas das éguas paridas ficou abaixo da encontrada 
Tabela 2 - Índices de perdas de prenhez de acordo com o status reprodutivo.

\begin{tabular}{llll}
\hline Status reprodutivo & Número de éguas & Número de perdas & $\%$ \\
\hline Falhadas & 18 & $1 / 18$ & $5,56^{\mathrm{a}}$ \\
Potrancas & 58 & $3 / 58$ & $5,17^{\mathrm{a}}$ \\
Paridas & 369 & $34 / 369$ & $9,21^{\mathrm{a}}$ \\
Total & 445 & $38 / 445$ & 8,53 \\
\hline
\end{tabular}

$\left.{ }^{(}\right)$letras iguais indicam que não houve diferença significativa ( $\mathrm{P}>$ $0,05)$.

por WOODS et al. (1987) de 14\%, não se evidenciando, contudo, um efeito benéfico das lavagens uterinas e aplicações de oxitocina. No trabalho de CHEVALIER-CLÉMENT (1989), foi observado índice de perdas de $6,5 \%$, sem a adoção da referida metodologia. Referente às éguas falhadas, o índice de perdas de 5,56\% (1/18) é bem inferior ao relatado por WOODS et al. (1987) de $14 \%(19 / 139)$ para éguas neste mesmo status reprodutivo. Esses resultados podem ser atribuídos ao fato de terem esses autores trabalhado com animais considerados inférteis por duas ou mais estações de monta, bem como por tratar-se de éguas de diversas raças e origens, ao contrário dos animais utilizados no presente estudo. Também não se observaram diferenças significativas $(\mathrm{P}>0,05)$ entre os índices de perdas de prenhez verificados no cio do potro e no cio subseqüente (Tabela 3), que estão abaixo daqueles relatados por WOODS et al. (1987) de $11 \%$ e $12 \%$, respectivamente. Esse resultado ratifica as condições adequadas de fertilidade em que se encontravam as éguas paridas utilizadas no presente trabalho, quando das inseminações efetuadas no cio do potro.

Pode-se verificar na tabela 4 que os índices de perdas em função das diferentes faixas etárias não diferiram estatisticamente $(\mathrm{P}>0,05)$. Contudo, evidenciou-se uma tendência dos indivíduos acima de 12 anos a terem fertilidade mais baixa, quando comparadas as perdas desta faixa etária com as demais. Este achado concorda com o reportado por WOODS et al. (1987) que, após

Tabela 3 - Índices de perdas de prenhez de acordo com a classificação do cio.

\begin{tabular}{llll}
\hline Classificação do cio & Número de éguas & Número de perdas & $\%$ \\
\hline Cio do potro & 258 & 23 & $8,91^{\mathrm{a}}$ \\
Cio subseqüente & 111 & 11 & $9,90^{\mathrm{a}}$ \\
Total & 369 & 34 & 9,21 \\
\hline
\end{tabular}

${ }^{(a)}$ letras iguais indicam que não houve diferença significativa (P > $0,05)$.
Tabela 4 - Índices de perdas de prenhez por faixas etárias.

\begin{tabular}{lccr}
\hline $\begin{array}{c}\text { Faixas etárias } \\
\text { (anos) }\end{array}$ & Número de éguas & Número de perdas & $\%$ \\
\hline $3-5$ & 115 & 8 & $6,95^{\mathrm{a}}$ \\
$6-8$ & 148 & 12 & $8,10^{\mathrm{a}}$ \\
$9-11$ & 127 & 10 & $7,87^{\mathrm{a}}$ \\
$>12$ & 55 & 8 & $14,54^{\mathrm{a}}$ \\
Total & 445 & 38 & 8,53 \\
\hline
\end{tabular}

${ }^{(a)}$ letras iguais indicam que não houve diferença significativa (P $>0,05$ ).

levantamento efetuado em vários haras, constataram uma elevação direta e contínua de perdas de prenhez em éguas acima de 13 anos. Estudos em humanos indicam que fatores intrínsecos, como anomalias cromossômicas, parecem incrementar-se com o aumento da idade (BOUIE \& BOUIE, 1976). De acordo com WOODS et al. (1987) e BALL (1993) o mesmo deve ocorrer em eqüinos, de tal modo que quanto mais velha a égua, maior a chance desses fatores causarem a perda da prenhez.

\section{CONCLUSÃO}

Os índices de perda de prenhez verificados neste estudo são semelhantes aos reportados na literatura, indicando um período de maior suscetibilidade entre a segunda e terceira semanas de gestação.

\section{FONTES DE AQUISIÇÃO}

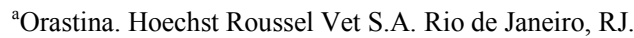

\section{REFERÊNCIAS BIBLIOGRÁFICAS}

BALL, B.A. Embrionic death in mares. In: McKINNON, A.O., VOSS, J.L. Equine reproduction. Philadelphia : Lea \& Febiger, 1993. Cap. 61. p.517-531.

BAll, B.A., LitTle, T.V., HILlMAN, R.B., et al. Pregnancy rates at days 2 and 14 and estimated embryonic loss rates prior to day 14 in normal and subfertile mares. Theriogenology, v.26, p.611-619, 1986.

BALL, B.A., WOODS, G.L. Embryonic loss and early pregnancy loss in the mare. Compendium on Continuing Education, v.9, p.459-470, 1987.

BLUE, M.G. A citogenetical study of prenatal loss in the mare. Theriogenology, v.15, p.295-309, 1981.

BOUIE, J.G., BOUIE, A. Chromosome anomalies in early spontaneous abortion: their consequences on early embryogenesis. Current Topics in Pathology, v.62, p.193208, 1976 
CHEVALIER-CLÉMENT, F. Pregnancy loss in the mare. Animal Reproduction Science, v.20, p.231-244, 1989.

DOUGLAS, R.H., BURNS, P.J., HERSHMAN, L. Physiological and commercial parameters for producing progeny from subfertile mares by embryo transfer. Equine Veterinary Journal, Suppl. 3, p.111-114, 1985.

DU PLESSIS, J.L. Some observations and data in Thoroughbred breeding. Journal of South African Veterinary Medical Association, v.35, p.215-221, 1964.

FERREIRA, J.B.P., PINHO, T.G., SANTOS, M.R.C., et al. Incidência e caracterização ultra-sonográfica da morte embrionária em éguas da raça campolina. Revista Brasileira de Reprodução Animal, v.23, p.202- 203, 1999.

GINTHER, O.J., GARCIA, M.C., BERGFELT, D.R. Embryonic loss in mares: Pregnancy rate, length of interovulatory intervals and progesterone concentrations associated with loss during days 11 to15. Theriogenology, v.24, p.409-417, 1985.
LOPES, M.D., PAPA, F.O., PRESTES, N.C., et al. Morte embrionária precoce em éguas: Aspectos clínicos e hormonais. In: CONGRESSO BRASILEIRO DE REPRODUÇÃO ANIMAL, 10., 1993, Belo Horizonte. Anais... Belo Horizonte : CBRA, 1993. v.2, p.231-380.

McKINNON, A.O., SQUIRES, E.L., PICKETT, B.W. Equine reproductive ultrasonography. Colorado State University. Animal Reproduction Laboratory. 1988. p.37-40. (Bulletin n.4).

PALMER, E., DRIANCOURT, M.A. Use of ultrasonic echography in equine gynecology. Theriogenology, v.13, p.203-216, 1980.

WOODS, G.L., BAKER, C.B., BALDWIN, J.L., et al. Early pregnancy loss in broodmares. Journal of Reproduction and Fertility, Suppl. 35, p.455-459, 1987. 